\title{
Advances in Auxiliary Power Unit Development For Dual Supply UAVs
}

\author{
Wasantha Samarathunga $^{1 *}$, Hideomi Arai ${ }^{2}$, Kenta Asaishi $^{3}$, Ken Ichiryu ${ }^{4}$
}

\begin{abstract}
This paper address recent developments on auxiliary power unit for dual supply unmanned aerial vehicles. The proposed APU is designed and developed as a scalable backup power solution to tethered UAV systems. The APU addressed in this paper consist of off-line battery power supply and a high speed fault detection and switching unit, to control the proposed APU. Target operation of APU is to switch the battery power whenever the primary cable power is unavailable and switch back to cable power whenever the cable power is available during the UAV flight mission. The switching speed of newly developed prototype in this research with high speed logic ICs and n-channel MOSFETs is proved to be within milliseconds range and therefore could be considered fast enough for low altitude hovering missions. The outcome of the proposed design is also compact and light weighted, hence making the proposed prototype applicable in many existing battery powered UAVs for hybrid use.
\end{abstract}

Index Terms-Auxiliary Power Unit, Tethered UAV, Switching Supply Power During Flight, Dual Supply UAV.

\section{INTRODUCTION}

Dual power supply is the option where a very single power supply is under uncertainties of operation. In case of tethered unmanned aerial vehicles the risk of power failure increases near payload thresholds. There are recent researches on developing backup battery power for UAVs. This research address on recent developments of auxiliary power unit (APU) design and its performance evaluation. Tethered UAVs are popular for flight missions with long hovering time and heavy payloads.

When it comes to design tethered power supply it is a requisite to use high voltages to avoid power loss during transmission. High to low voltage conversion is done on aerial vehicle before run the motors. Tethered UAVs operate less than 400VDC is quite popular. UAV motors normally run on $48 \mathrm{VDC}$ in heavy payload vehicles or in light payload vehicles $24 \mathrm{VDC}$. In order to make the available payload high, things mounted on vehicle should be as lighter as possible. The same rule applies to power conversion unit, which explains usage of light weighted switching regulators as DCDC converters. Figure 1 illustrates the tethered UAV system used in this research.

Wasantha Samarathunga ${ }^{1^{*}}$, MONOZUKURI Mechatronics Laboratory, 2161-12 Miyama-cho, Hachioji, Tokyo Japan.

Hideomi Arai $^{2}$, MONOZUKURI Mechatronics Laboratory, 2161-12 Miyama-cho, Hachioji, Tokyo Japan.

Kenta Asaishi ${ }^{3}$, MONOZUKURI Mechatronics Laboratory, 2161-12 Miyama-cho, Hachioji, Tokyo Japan.

Ken Ichiryu', MONOZUKURI Mechatronics Laboratory, 2161-12 Miyama-cho, Hachioji, Tokyo Japan.
Switching regulators are nonlinear power supply compares to batteries. In other words batteries are the most reliable power source. Recent developments on UAV batteries are explained in [2, 3 and 4]. Due to required power density to operate tethered UAVs, paralleling switching regulators are necessity. This normally add complexities and bring risks to the system, despite of finite requirement to do so. To avoid risks and critical failures it is necessary to implement APUs on tethered aerial vehicles.

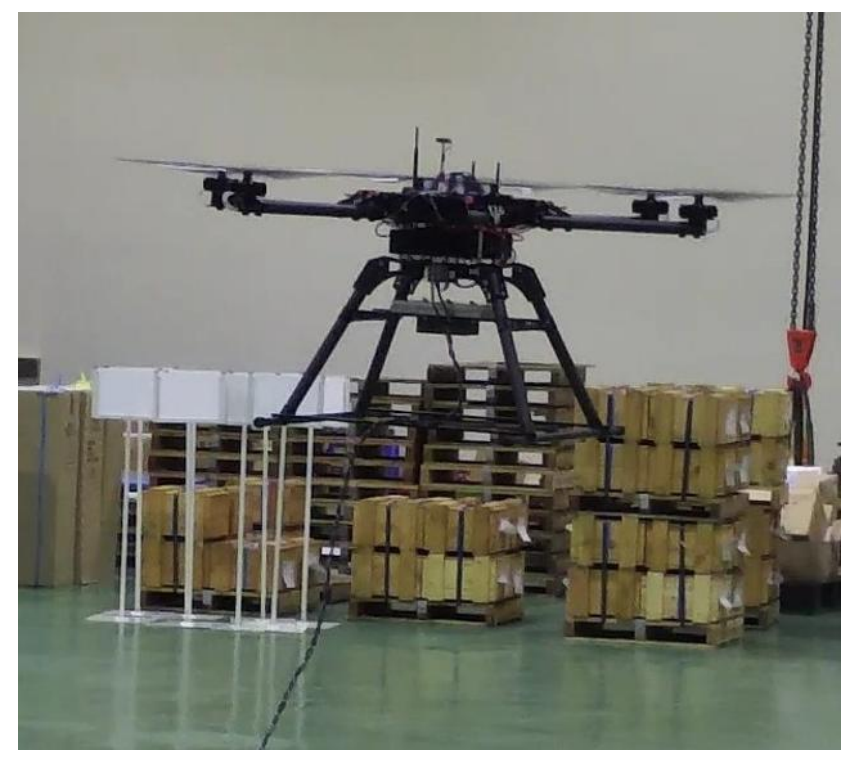

Figure 1: Tethered UAV System

The following are common APU unit types,

(1)On-line prototypes

Denotes prototypes with simultaneous charging and power supplying capability.

(2) Hybrid prototypes

Denotes prototypes that capable of charging when input power is available and discharge the battery when a power failure is detected.

\section{(3) Off-line prototypes}

Denotes prototypes equipped with pre-charged batteries and activate the discharge with detected power failure.

Off-line APU are considered to be more scalable than on-line and hybrid APUs, thus became more attractive for research and developments. In this research we introduce new APU prototype and make comparison with a conventional prototype which uses solid state relay (SSR) as switching device. Conventional APU which subject to the comparison denotes SSR based APU in this paper. As illustrated in [1] SSRs are highly attractive in power switching applications 
nowadays.

Even though it is technically safe and simple to proceed with SSR as a switching device, it has certain considerations on size, weight and response time. Therefore in this paper a new prototype is proposed and tested. This research aims to evolve the SSR based APU prototypes into a different group.

Expected advantages of the proposed design are,

(1)Faster high power switching.

(2)Minimum black out time, less or no turbulence during switching.

(3)The switching device will be much smaller and light weighted. This in turn bring further advantages as increase payload capacity and expandability of small battery based UAVs toward hybrid types.

Organization of the rest of the paper is as follows. In the very next section of this paper it is explained the philosophical concept to support our engineering design. Then in section 3, SSR based APU is introduced. Section 4 is about the proposed APU and section 5 is about switching tests to obtain switching characteristics. Then based on test results we consider whether the switching speed could be improved. Then finally we draw conclusions in section 6 upon comparing test result of both new and SSR based APUs.

\section{PHILOSOPHICAL CONCEPTS IN ENGINEERING DESIGN}

The logical argument on this engineering development is as follows. Firstly it starts with strong assumption, i.e. philosophical term "apriori" is applicable here. With that the proposed design is both consist and fastest to achieve the proposed goal. If there exists another methodology which could perform better than the former, then former has to be replaced with latter till even better methodology will be introduced. If no such a methodology is presence then the first "apriori "stays valid.

\section{SSR BASED APU}

The following figure 2 illustrates a SSR based APU. Figure 3 illustrates the components of control circuit and in figure 4 illustrates switching speed characteristics of SSR based APU.

Figure 4 shows that during cable to battery power switching a 0.25 second delay is presence. This delay approximates a $20 \mathrm{~cm}$ vertical drop (in other words minor turbulence) for the UAV.

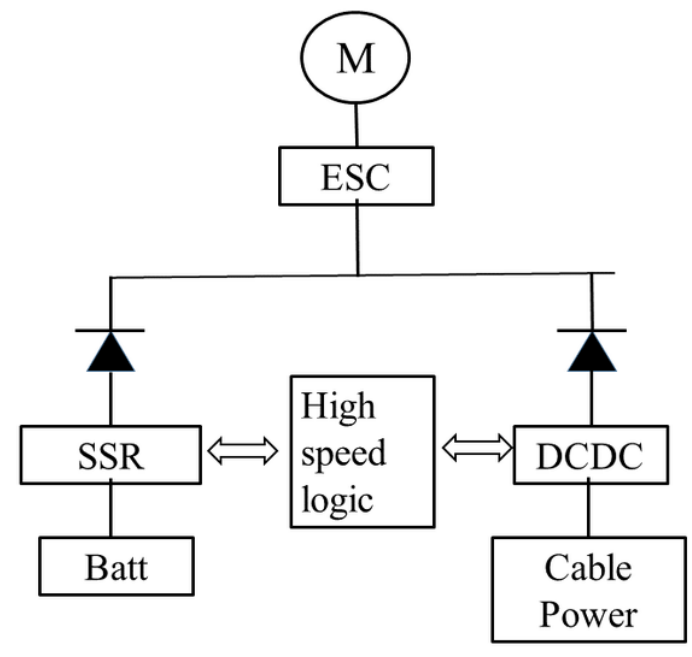

Figure 2: SSR based APU

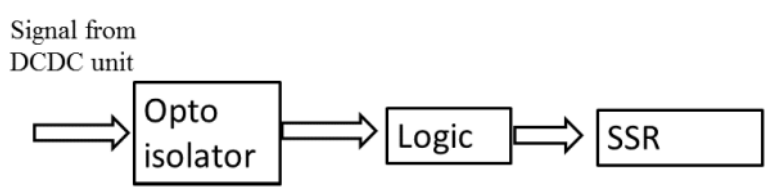

Figure 3: Components of Control Circuit (SSR based APU)

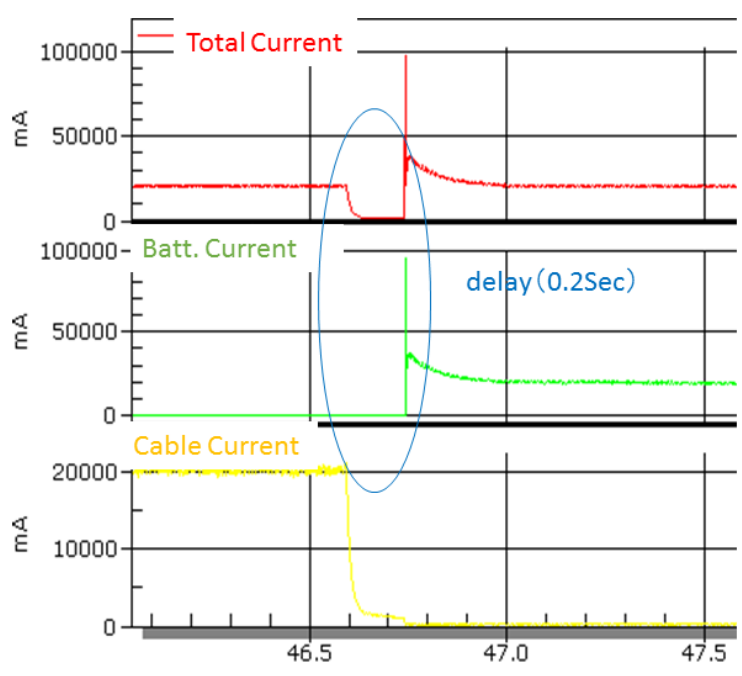

Switch from Cable Power to Battery Power

Figure 4: Switching Speed of SSR based APU

\section{THE PROPOSED DESIGN}

In the proposed prototype switching device SSR is replaced with MOSFETs and control circuit is also modified. Since we need to switch high currents, $\mathrm{n}$-channel MOSFET is considered with low side switching. Selection is subject to availability. Since n-channel MOSFETs are used it is necessary to produce separate gate pulse less than 20VDC which in turn is controlled by NPN-PNP compound with 5 VDC logic output. Following figure 5 illustrates the new 
prototype and figure 6 illustrates control circuit components.

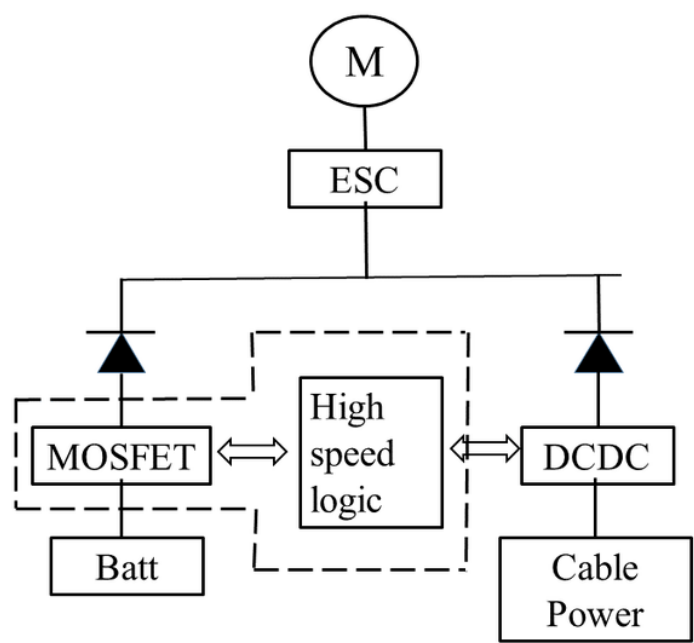

Figure 5: New Prototype

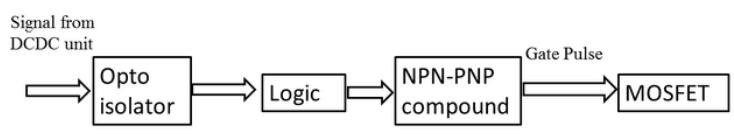

Figure 6: Components of Control Circuit

\section{SWITCHING SPEED EVALUATIONS}

The following figures illustrates the switching speed characteristics of the proposed APU. Figure 7 is the full test with assumed power fail during cable power flight, i.e. switching sequence is cable to battery and battery to cable. Figure 8 illustrates in detail the cable to battery switching.

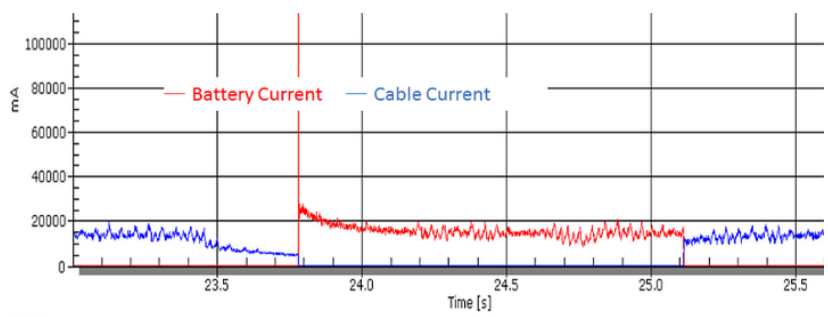

Fig 7: Switching Test

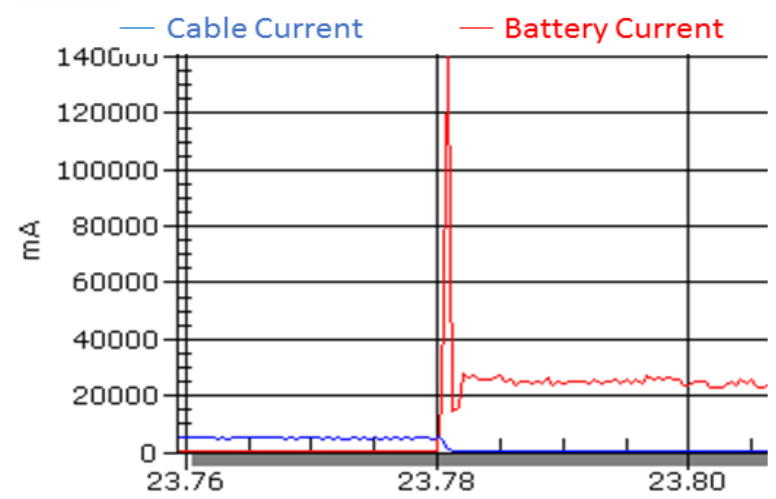

Fig 8: Cable to Battery Switching
Following figures illustrates the input and output control signals of APU. Figure 9 shows the control signals during the test and figure 10 illustrates the control signals during cable to battery switching.

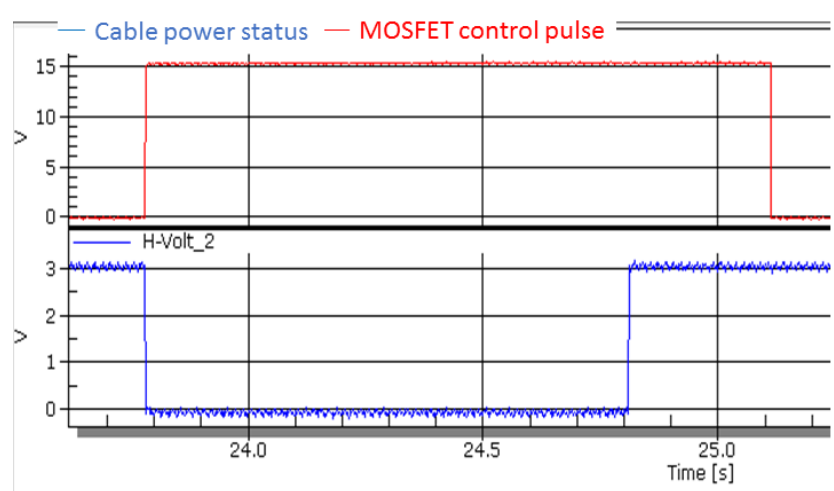

Fig 9: Control Signals of APU

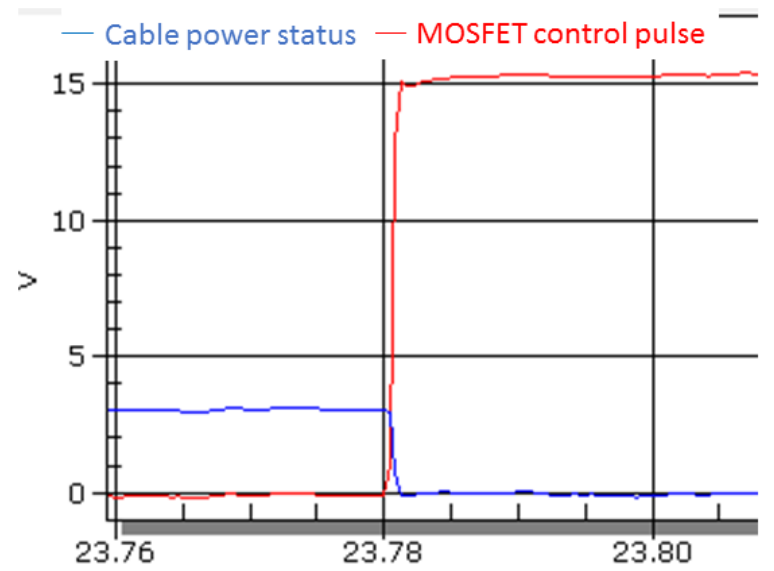

Fig 10: Control Signal during Cable to Battery Switching

\section{CONCLUSION}

From the test results above, it can be expressed that the proposed APU is faster than SSR based APU. Comparing the results from figure 4 vs figure 8 and 10 this switching speed improvement is apparent, thus switching speed reached to milliseconds range.

In this research a tethered UAV is used where primary power is cable power and the monitored signals are auxiliary power from DCDC conversion units. The conclusive point here is that if the monitored signals are fast enough even if the primary source is different, this APU could perform equally well. Hence this APU could be applicable in general dual supply problems.

\section{REFERENCES}

[1] W. Kuang, S.W. Or, C.M. Leung and S.L. Ho, Development of piezoelectric Transformer-Coupled Solid State Relay for Electrical Circuit Control in Railway Systems, Proceedings of the 1st International Workshop on High-Speed and Intercity Railways. Voulme 2, ISBN 978-3-642-27962-1, pp328-338.

[2] Magdalena Dudek, Piotr Tomczyk, Piotr Wygonik, Mariusz Korkosz, Piotr Bogusz, Bartomiej Lis, "Hybrid Fuel Cell ?Battery System as a Main Power Unit for Small Unmanned Aerial Vehicles (UAV)", 
International Journal of Electrochemical Science, (ISSN 1452-3981) vol.8, 2013, pp8442-8463.

[3] "High-Power Lithium Technology Proves Ripe For Military", A press release, Available at:

http://www.powerfactor.co.uk/announcements/high-power-lithium-tec hnology-proves-ripe- for-military-applications", Posted: 17-Jul-2013.

[4] "Horizon launches Hycopter fuel cell multirotor UAV", Volume 2015 Issue 6, June 2015, pp-4, doi:10.1016/S1464-2859(15)30145-0, Available at:

http://www.sciencedirect.com/science/article/pii/S1464285915301450 